\title{
COMPLETE REMOVAL OF THREE VERTEBRAE FOR GIANT-CELL TUMOUR
}

\author{
Bertil Stener, Göteborg, Sweden, and Odd E. Johnsen, Skien, Norway
}

Removal of the whole of three adjacent vertebrae, largely destroyed by tumour, does not appear to have been reported hitherto. Such an operation was carried out on May 15, 1968, at the Neurosurgical Department (Head: Professor Tormod Hauge) of the Rikshospitalet, Oslo, Norway. A paper concerning this operation was presented at the Memorial Centre for Cancer and Allied Diseases in New York City (June 1968) and at the International Symposium on Operative Treatment of Bone Tumours in Basle (January 1969).

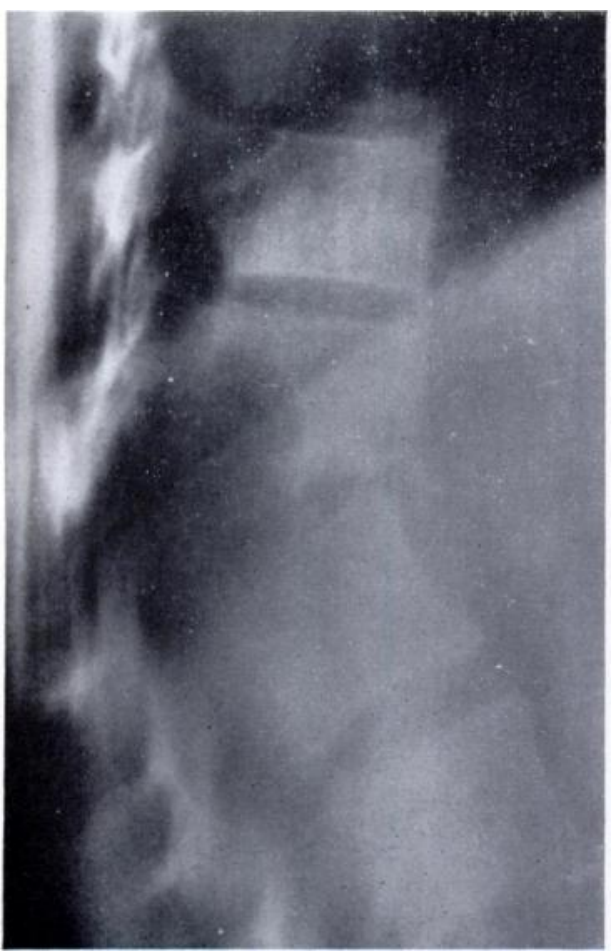

FIG. 1

Sagittal tomograph. The body of the twelfth and the bodies of the eleventh thoracic and first lumbar vertebrae have been partially destroyed. There is sharp angulation where the body of the eleventh thoracic vertebra rides on the anterosuperior edge of the body of the first lumbar vertebra. thoracic vertebra has been completely destroyed

\section{CASE REPORT}

A twenty-six-year-old woman began to suffer pain in the thoraco-lumbar region in January 1967. Just over a year later, in February 1968, she gave birth to her third child. About a week after delivery she began to notice increasing weakness in both legs, and by March 10 she was unable to walk. At the beginning of April she was admitted to the Neurosurgical Department of the Rikshospitalet in Oslo with a suspected intraspinal tumour. The patient was then almost paralysed in both lower extremities; only slight movement of the toes was possible. Sensibility to touch and pain was much reduced in the area below the hip joints. No reflexes could be elicited from the lower third of the abdominal wall. On both sides the knee jerk was positive, the ankle jerk exaggerated and the Babinski sign absent. The patient had lost control of the bladder.

Radiographs of the spine showed angulation at the level of the twelfth thoracic vertebra (Fig. 1). The body of this vertebra had been destroyed by an osteolytic process which also involved the postero-inferior part of the body of the eleventh thoracic vertebra and the postero-superior part of the body of the first lumbar vertebra.

Angiography (Figs. 2 to 5) demonstrated that the aorta was displaced forward and to the left at the level of the skeletal destruction. In a later phase of the angiography a rounded, well delineated mass, measuring 10 by 10 by 7 centimetres, became opaque with contrast medium, presumably because of the filling of abundant fine vessels. The centre of this mass was located near the point where the bodies of the eleventh thoracic and first lumbar vertebrae met anteriorly. The findings suggested that a highly vascular tumour had destroyed the body of the twelfth thoracic vertebra and then expanded in all directions, invading the bodies of the eleventh thoracic and first lumbar vertebrae. Obviously the displacement of the aorta 


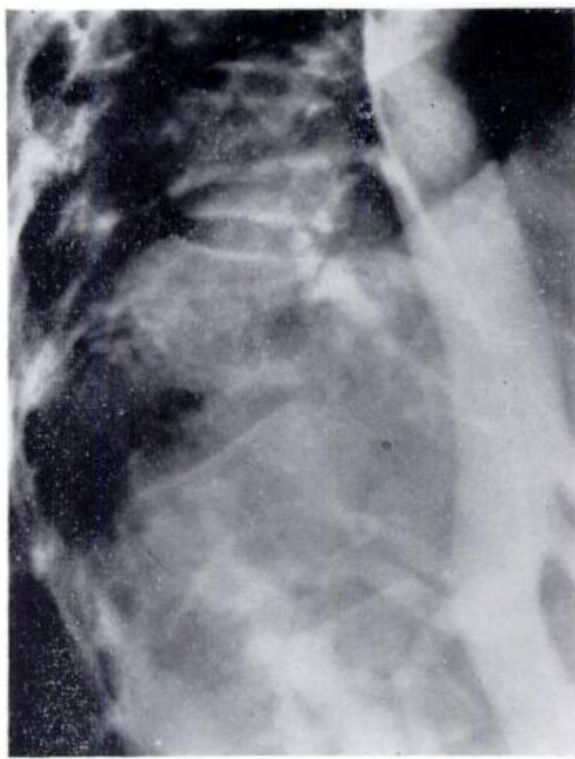

FIG. 2

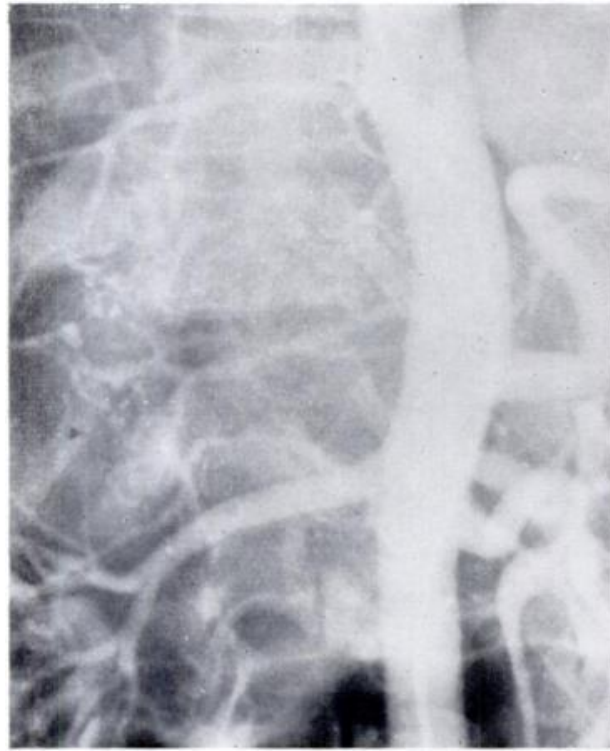

FIG. 4

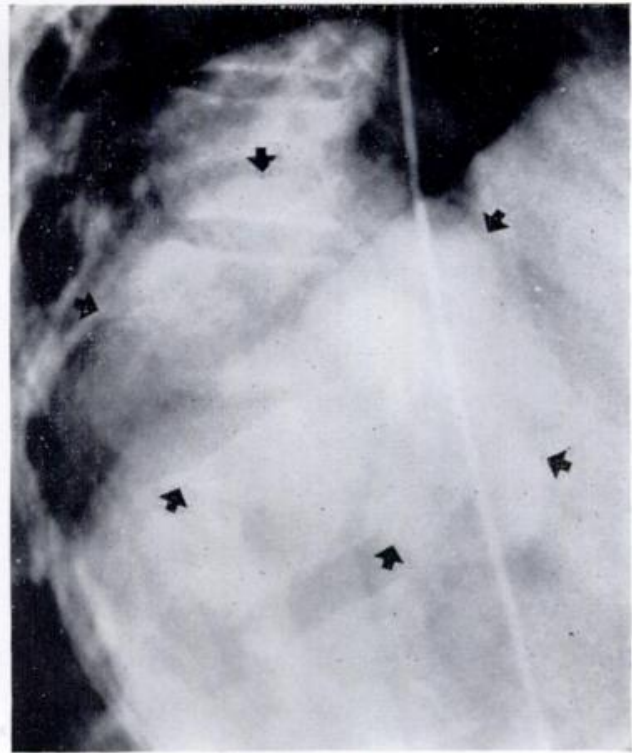

FIG. 3

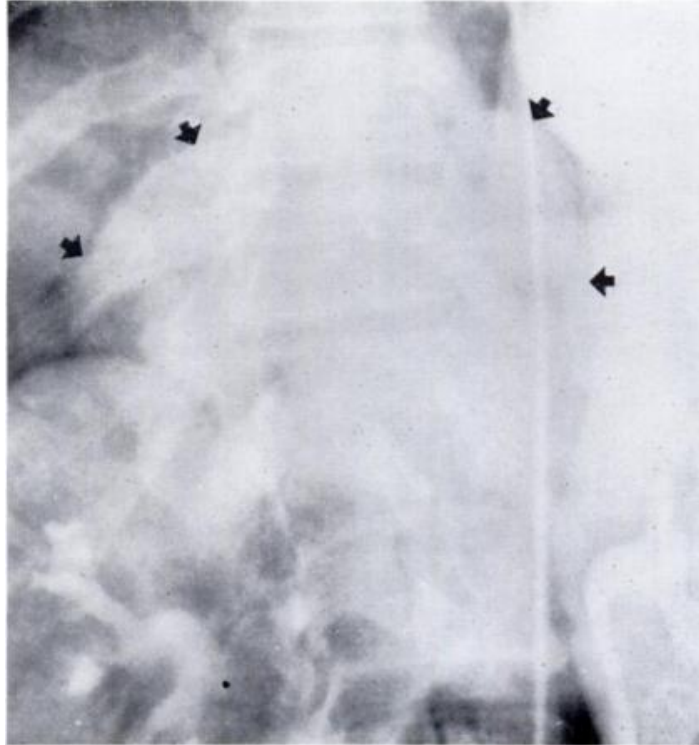

FIG. 5

Angiography. Figure 2-Lateral view, early phase. The aorta is displaced forward in a smooth curve at the level of angulation. Figure 3-Later phase. The tumour (encircled by arrow's) has become perfused with contrast medium. Figure 4-Antero-posterior view, early phase. The aorta is displaced to the left by the tumour. Figure 5-Later phase. The tumour has become visible because of filling with contrast medium. The latero-superior contours are marked by arrows. The inferior contour is not clearly distinguishable in this view. 
was caused by the expanding tumour (Fig. 6). Myelography was performed but gave no useful information.

Laminectomy-In order to decompress the spinal cord, the laminae with the spinous processes were removed from the eleventh and twelfth thoracic and the first lumbar vertebrae; a small piece of tumour tissue was excised for microscopy from the site of the destroyed left pedicle of the twelfth thoracic vertebra. Only slight improvement of the function of the spinal cord was noted after the laminectomy; the patient remained without control of the bladder. The histological report indicated a benign giant-cell tumour of bone.

One month after the laminectomy radiography of the spine showed increased skeletal destruction (Fig. 7), and contrast medium remaining from the myelography was blocked at the level of the involved vertebrae. It was obvious that a disastrous development was to be

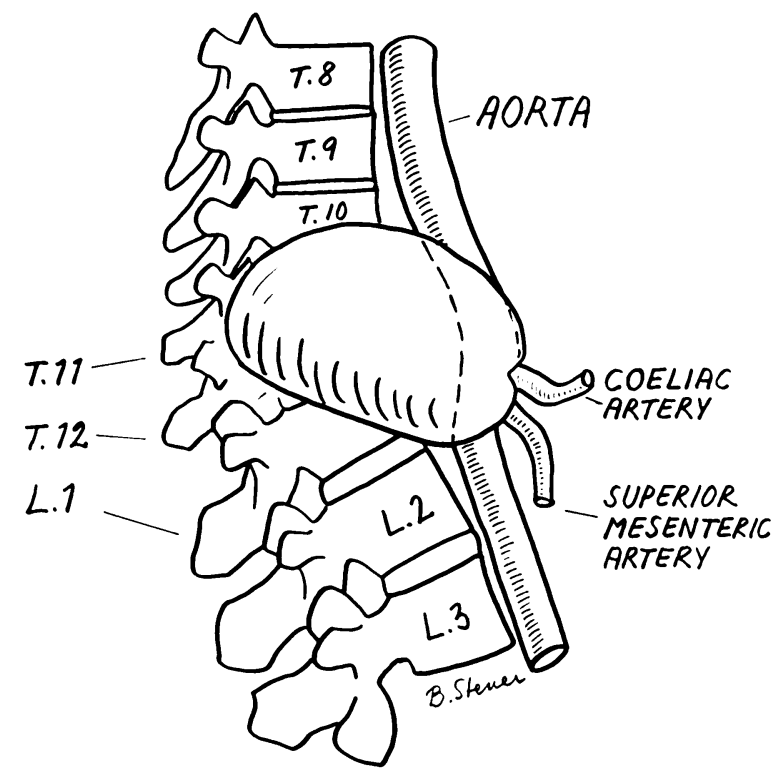

Fig. 6

Synopsis of the radiographic information. A highly vascular tumour has destroyed the body of the twelfth thoracic vertebra completely and the bodies of the eleventh thoracic and first lumbar vertebrae partially. The tumour bulges into both pleural cavities, and displaces the aorta forward and to the left at the origin of the coeliac and superior mesenteric arteries.

expected if the tumour were allowed to continue its course. Radiotherapy was considered, but in agreement with several authorities the radiotherapist declined such treatment unless the tumour was inaccessible to operation. Originally the tumour had been deemed inoperable, but after review of the radiographic findings it was thought that possibly it could be extirpated by complete removal of the three vertebrae involved. Therefore, after discussion with the patient and her husband, operation was decided upon. In the planning of the operation the angiographic information about the extent and topography of the tumour was most helpful. Operation-The patient was placed in the prone position with the chest and the pelvis each supported by two cushions, one on either side; pressure against the inferior vena cava was avoided in order to minimise bleeding from vertebral and paravertebral veins (Relton and Hall 1967). A posterior midline incision circumscribing the laminectomy scar was made from the spinous process of the fifth thoracic vertebra to the sacrum. At the top and the bottom the incision was prolonged to either side: above in the latero-superior direction for 6 centimetres, 
below in the latero-inferior direction for 9 centimetres. Then a large flap of skin, subcutaneous fat and superficial fascia was raised on either side, exposing the trapezius and the latissimus dorsi. These muscles, with the underlying lumbar fascia, were severed from the spine and raised so that the erector spinae muscles were exposed. At the thoraco-lumbar level each erector spinae muscle was divided transversely and retracted upwards and downwards. The lower half of the thoracic cage was now exposed. The twelfth and eleventh ribs were removed subperiosteally on either side, except for the heads and necks, which were left to be removed later along with the tumour. In addition, the tenth, ninth and eighth ribs were resected subperiosteally from their angles to the tips of the transverse processes. The quadratus lumborum and the upper parts of the psoas were severed from the spine on both sides.

Once the thoracic wall had been made soft and flexible by the resection of ribs, an extrapleural dissection was attempted. It had to be given up, however, because the parietal pleura was found to be firmly adherent to the postero-lateral parts of the tumour (Fig. 6). Instead, the pleural cavity was opened on both sides. The azygos and the hemiazygos veins were divided and ligated, and the aorta was exposed above and below the tumour. The attachments of the diaphragm to the anterior surface of the tumour were severed. A careful dissection was made in order to avoid damage to the splanchnic nerves and thoracic duct. The displaced aorta was firmly adherent to the tumour, and after some attempts to dissect it free it was judged safer to postpone this part of the operation until the involved vertebrae had been cut loose from the spine.

The tumour was enclosed in a capsule attached to the bodies of the eleventh thoracic and first lumbar vertebrae. Within this capsule the tumour bulged upwards past the disc between the tenth and eleventh vertebrae and downwards beyond the disc between the first and second lumbar vertebrae (Figs. 3, 5 and 6). These discs, however, although adjacent to the tumour, were not infiltrated and could be separated from the capsule of the tumour by blunt dissection. After this the thecal sac was dissected free posteriorly by removing scar tissue formed after the laminectomy. This part of the operation was facilitated by removal of the spinous process and most of the lamina of the tenth thoracic vertebra. In this way the dura was first exposed where it was free from adherent scar tissue; the dissection was then carried out cephalo-caudally. The eleventh and twelfth thoracic nerve roots were severed

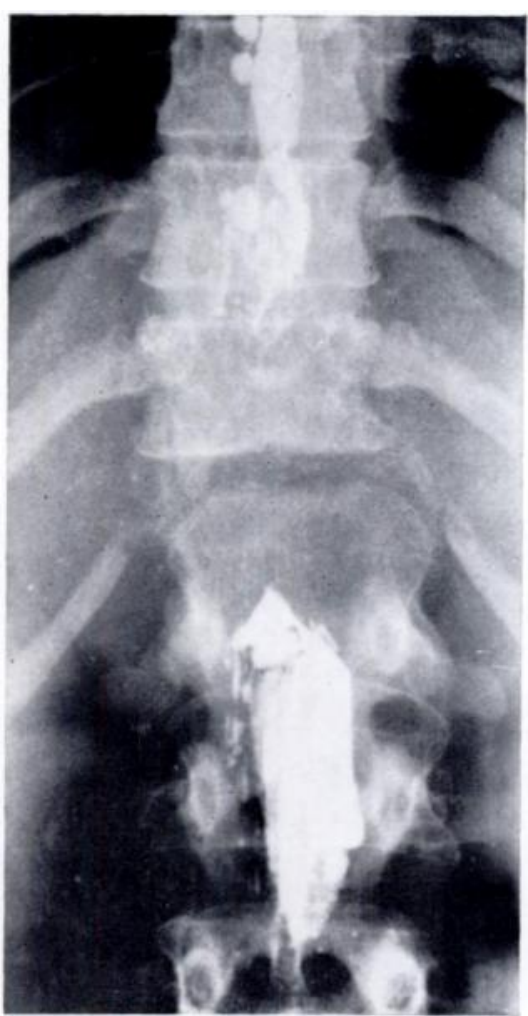

FIG. 7

Radiograph four weeks after myelography and subsequent laminectomy of eleventh and twelfth thoracic and first lumbar vertebrae. The radiopaque medium is blocked at the level of the involved vertebrae. The body of the eleventh thoracic vertebra appears less destroyed than it is because the projection is parallel to the superior and inferior surfaces of this vertebra; the body of the first lumbar vertebra appears more destroyed than it is because it is seen with the superior surface tilted backwards (compare Fig. 1). The twelfth ribs are hanging loose. and ligated close to the thecal sac. The first lumbar nerve roots were severed more peripherally, the object being to spare, if possible, arterial branches accompanying these roots to the spinal cord.

As soon as the thecal sac had been dissected free as far forward as possible from behind, the disc between the tenth and eleventh vertebrae and that between the first and second lumbar vertebrae were cut through. The tumour was then removed together with all residual parts

VOL. 53 B, NO. 2, MAY 1971 
of the eleventh and twelfth thoracic and the first lumbar vertebrae. Because it embraced the spinal cord the tumour had to be removed in several parts. However, before tumour tissue was entered, the aorta was occluded to reduce bleeding and the large wound was carefully

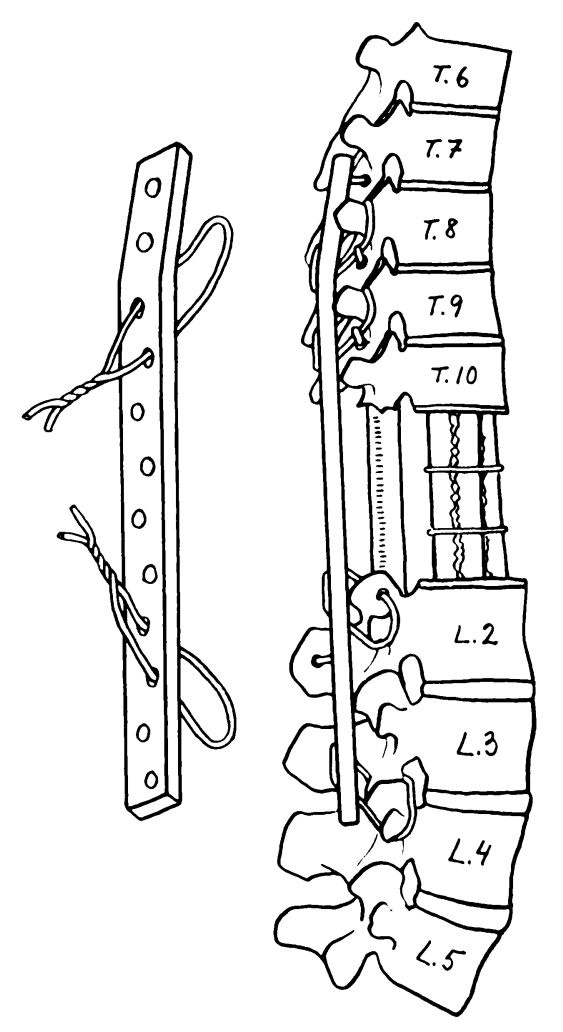

FIG. 8

Reconstruction of the spine (see text). draped with towels. After removal of the specimen the only structure bridging the gap between the tenth thoracic and the first lumbar vertebrae was the spinal cord enclosed in the thecal sac. The towels were removed and the occlusion of the aorta was released. A small hole in the aorta was sutured.

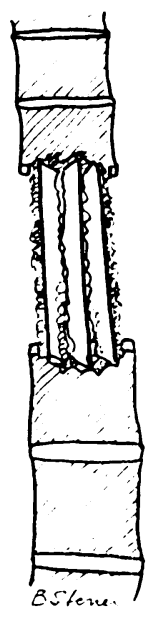

Reconstruction (Fig. 8)-The disc below the tenth thoracic vertebra and the disc above the second lumbar vertebra were removed except for a peripheral portion of the annulus fibrosus. The bony surfaces adjacent to the removed parts of the discs were chiselled away, exposing cancellous bone. Then, five struts of cortical bone from both tibiae, six centimetres long and one centimetre broad, were put together and inserted between the tenth thoracic and the second lumbar vertebrae. All five struts could be placed within the fibrous ring of the second lumbar vertebra, but only four of them within the smaller ring of the tenth thoracic; the bundle of struts was therefore encircled by two wires of stainless steel. Cancellous bone from the upper ends of the tibiae was put between and outside the cortical grafts. Then, in order to produce stability, two "A.O."* plates, measuring 20 by 1.6 by 0.6 centimetres and provided with twelve holes, were fixed by double steel wires to the spine above and below the gap (Fig. 8, centre). The plates, slightly bent so as to fit the thoracic kyphosis, were set in a frontal plane, one on each side of the spinous processes. In the thoracic region the wires were passed round the transverse processes of the eighth and ninth vertebrae. These processes are directed postero-laterally; therefore, in order to prevent slipping of the wires, those gripping the right and left transverse processes of one vertebra were fixed together by a wire passing through a bore-hole in the spinous process of the same vertebra. In the lumbar region, at a lower level, the wires were passed round the transverse process and the superior articular process of the fourth lumbar vertebra and the inferior articular process of the third lumbar vertebra, and at a higher level, round the transverse process and through the superior articular process of the second lumbar vertebra. To effect compression against the ends of the inserted cortical grafts the wires were passed through holes in the plate below the level of the skeletal gap in the thoracic region, and above the skeletal gap in the lumbar region (Fig. 8, left). By winding the wires tightly, satisfactory stability was achieved. Further fixation was then secured by joining one plate to the other by wires passed through bore-holes in the spinous processes of the seventh thoracic and second lumbar vertebrae. The reconstruction of the spine did not result in a complete alignment of the thoracic and lumbar vertebral columns in the

* The Swiss Association for Osteosynthesis. 
frontal plane. After the reconstruction the lumbar spine was displaced somewhat to the left in relation to the thoracic spine, as was the case before the operation (Fig. 7). This meant that the inserted cortical grafts deviated to some extent from bottom left to top right. No soft tissue was put round the thecal sac. Thus, after the reconstruction of the spine, free spaces. approximately one centimetre broad, separated the spinal cord from the grafted bone and the "A.O." plates (Fig. 8, centre). Suction tubes were inserted into both the pleural cavities. The erector spinae muscles were replaced into proper position and sutured; they then covered the metallic material. The trapezius and the latissimus dorsi were sutured in the midline, and finally, the skin flaps were sutured.

Histology-The consistency of the tumour was firm. The cut surface was grey with brown spots. The histological picture was that of a giant-cell tumour of bone (Fig. 9). The numerous giant cells contained many nuclei of uniform size with a marked nucleolus. The stromal cells were spindle shaped or polygonal, with oval or round nuclei. The cells were meagrely interspersed with collagenous fibrils. Occasional stromal cells presented evidence of mitotic division, but no apparent atypism was present. In a few areas the stroma contained fine trabeculae of osteoid tissue and bone.

Progress-During the first few days after the operation the patient had difficulty with breathing. and radiographs revealed atelectasis of the middle lobe of the right lung. However, the patient was helped successfully over this period by tracheal suction and assisted coughing.

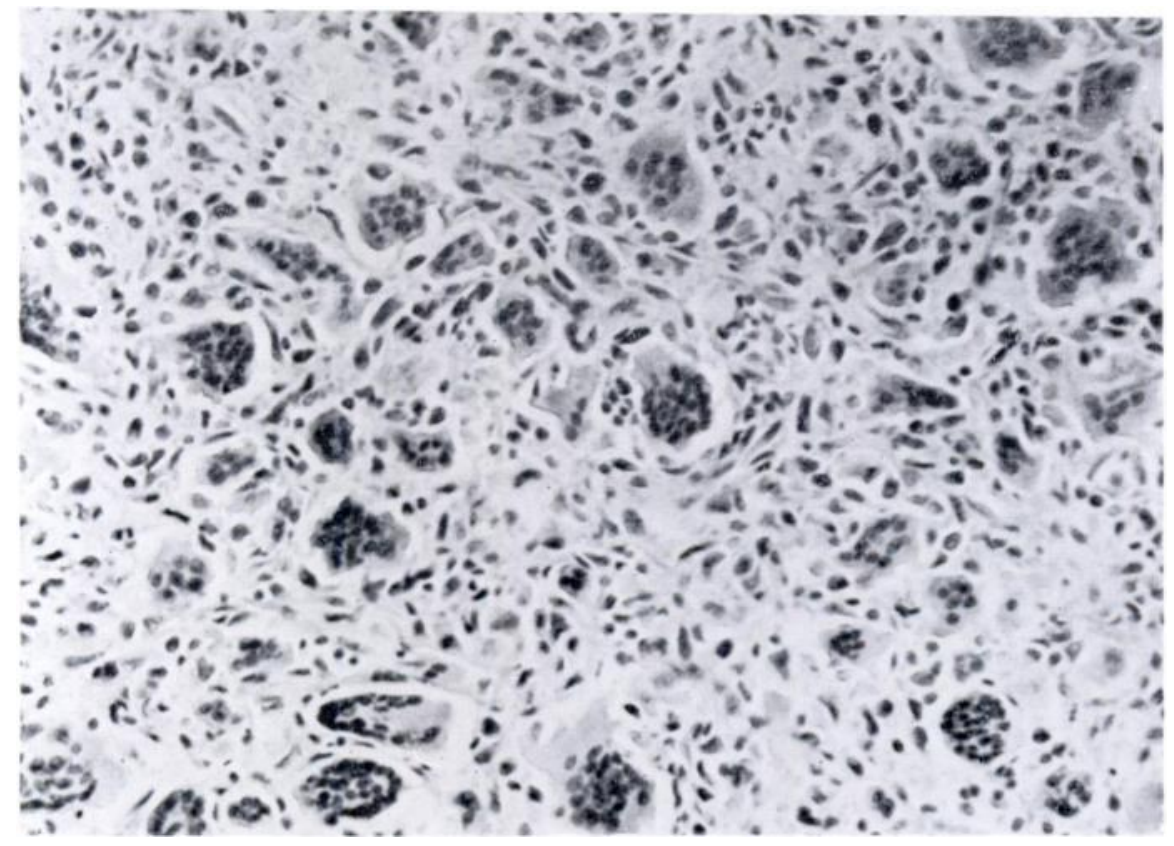

FiG. 9

Photomicrograph of the tumour. (Haematoxylin and eosin, $\because 230$.)

External fixation of the patient was not used: we felt that the stability achieved was good enough to make the use of a plaster bed unnecessary. In the first days after operation the patient was turned carefully to one or the other side at regular intervals. From about one month after the operation a "circle bed" (Stryker Circolectric) was used to facilitate turning of the patient.

Within a few hours of the operation perception of touch in the legs was already improved and she could move both feet. Within a few weeks sensibility became virtually normal, and within a few months she regained power to move all joints of the lower limbs. A few weeks

VOL. 53 B, NO. 2, MAY 1971 


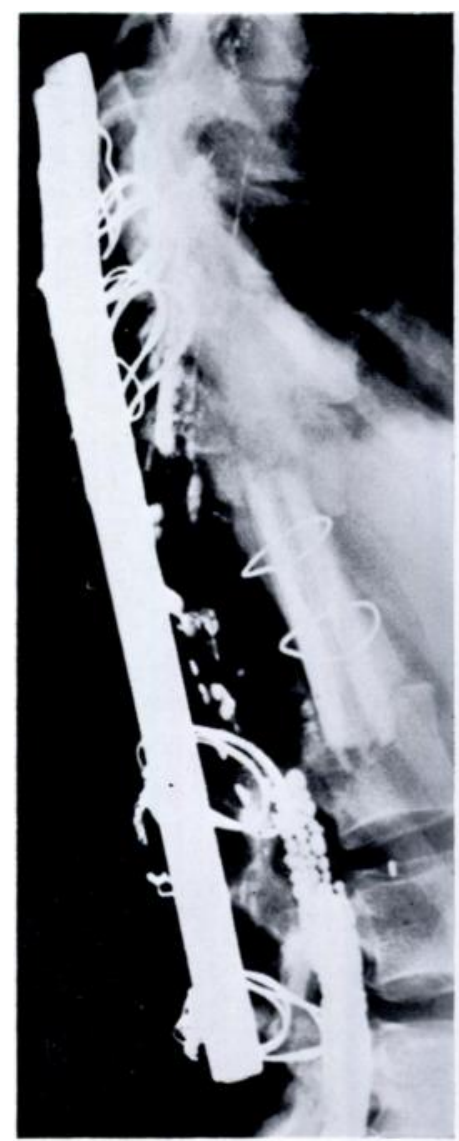

Fig. 10

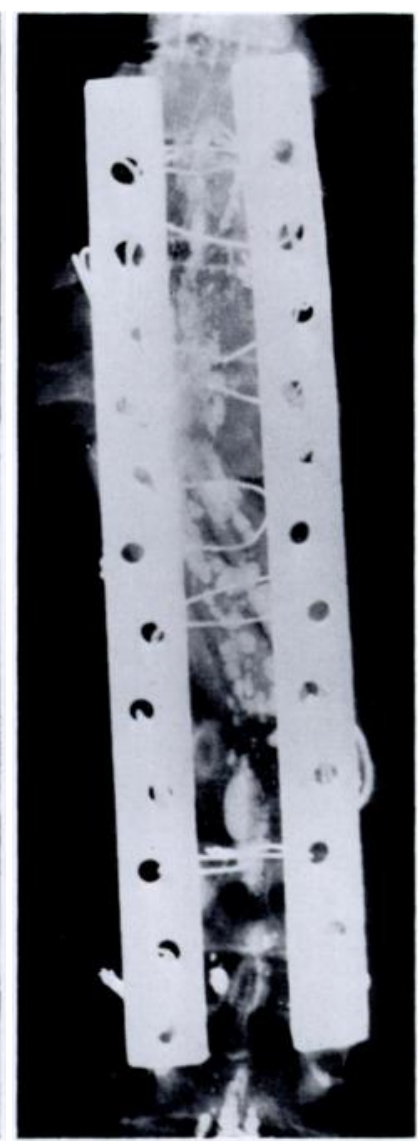

Fig. 11

Radiographs ten weeks after operation. Figure 10-Lateral view. Evidence of callus formation adjacent to the cortical grafts at the lumbar junction. Figure 11-Antero-posterior view.

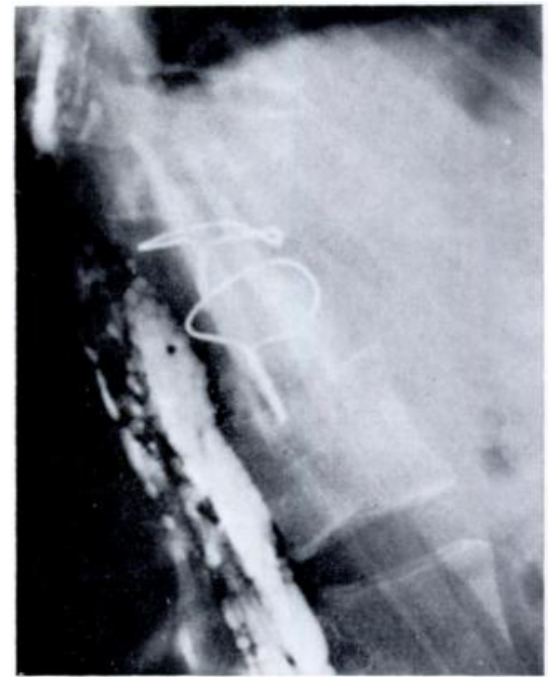

FIG. 12

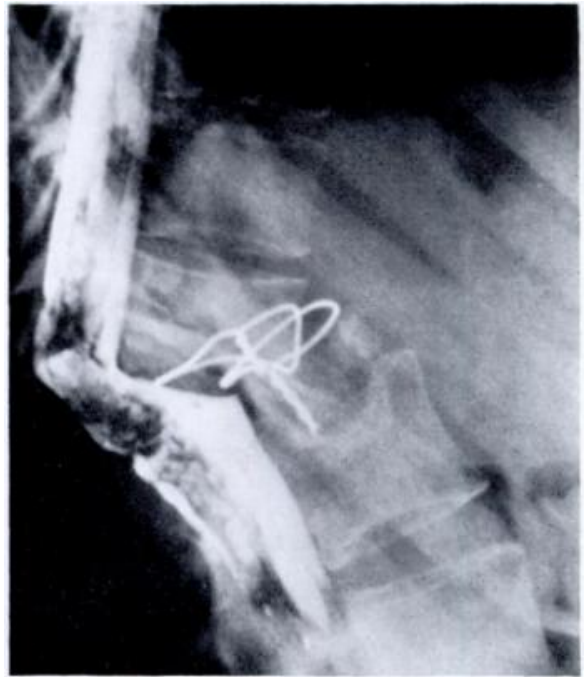

FIG. 13

Figure 12-Radiograph six months after operation. The plates and wires have been removed. Figure 13-Nine months after operation. The cortical grafts have been largely resorbed Approximation of the tenth thoracic and second lumbar vertebrae. 
after the operation the patient sensed filling of the bladder, and after six weeks the catheter was removed. Since that time she has had full control of the bladder.

Radiographs ten weeks after the operation showed callus adjacent to the grafted bone. obvious at the lumbar junction but less so at the thoracic junction (Figs. 10 and 11). The deviation of the cortical grafts in the frontal plane had increased since operation, but not as compared with a radiograph taken seven weeks after the operation. The increased frontal deviation of the grafts was accompanied by some angulation forward between the grafts and the thoracic spine.

In September 1968 the patient was transferred to the Department of Orthopaedic Surgery, Telemark Sentralsjukehus, Skien, Norway. Two weeks earlier serous fluid had started to drain from a sinus that had opened in the middle of the scar. This complication was thought to be due to a foreign-body reaction against the inserted metallic material. Five months after the operation, when the "A.O." plates felt loose and apparently no longer had any stabilising function, it was decided to remove them along with the wires. This was done in October 1968. During the operation it was found that the sinus had no communication with the grafted bone. Granulation tissue that had formed round the plates and wires was also removed. The wound was sutured and healed primarily. Figure 12 shows a radiograph taken one month after this operation. Clinically the spine then appeared to be stable. However, in January 1969 radiographs showed progressive absorption of the cortical grafts and approximation of the bodies of the tenth thoracic and second lumbar vertebrae. In February, when the radiograph shown in Figure 13 was taken, a new sinus had opened in the scar. This was assumed to be caused by a foreign-body reaction against the remaining metallic material-the wires used for encircling the struts of cortical bone. A further operation was done: in March 1969,

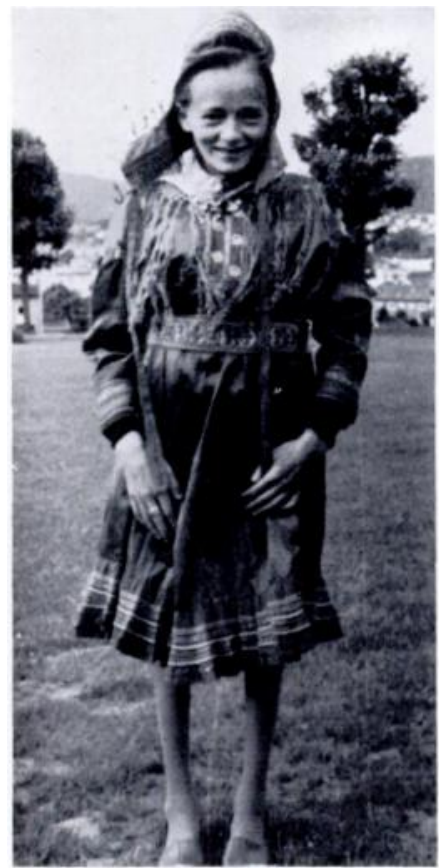

FIG. 14

Patient one year and six weeks after operation. through a dorso-lateral approach, the right side of the thoraco-lumbar region of the vertebral column was exposed. The wires were removed as were also some loose sclerotic pieces of bone, remnants of the cortical grafts, which were surrounded by granulation tissue. Inspection of the inferior part of the body of the tenth thoracic vertebra and the superior part of the body of the second lumbar vertebra did not reveal any sign of recurrent tumour. Several pieces of bone and soft tissue were taken for histological examination. This revealed granulation tissue with foreign-body reaction, but no tumour tissue. The wound healed primarily and remained closed thereafter. After the operation the patient was provided with an orthopaedic corset with struts.

In May 1969, one year after the removal of the tumour, the patient was allowed to start walking in a walking chair. In July the spine felt stable, and the patient could easily stand without support (Fig. 14). She had normal sensation in both lower extremities, and good strength in all muscle groups. The patellar and ankle reflexes were exaggerated (as were also the tendon reflexes of the upper extremities). The Babinski sign was not present. Function of the bladder and the bowel was normal.

At this time the patient had been away from her family for well over a year, and for psychological reasons it was deemed inadvisable to keep her any longer in the south of Norway because her husband, a Lapp, lived a nomadic life in the northern part of the country. The patient therefore was flown up to a hospital in Hammerfest, but her longing for the family was so great that she demanded to be transferred, at her own risk, to a cottage hospital near

VOL. 53 B, NO. 2, MAY 1971 
her husband's abode. There her general physical and psychological condition much improved and soon she was able to walk without support. Radiographic examination (Figs. 15 and 16) in Hammerfest eleven months after the last operation showed increased angulation in the sagittal plane, but no evidence of recurrent tumour. Sclerotic bone had formed at the junction between the bodies of the tenth thoracic and second lumbar vertebrae. The patient reported herself as being well and having improved in strength. When visiting her family she had even been able to throw the lasso which is used for catching reindeer.

\section{COMMENT}

The result of this operation is much better than we had expected. What we had hoped to achieve when deciding to operate was to arrest the progressive destruction of the spine and to save the patient to a life in a wheel-chair. The patient had been paraplegic and without

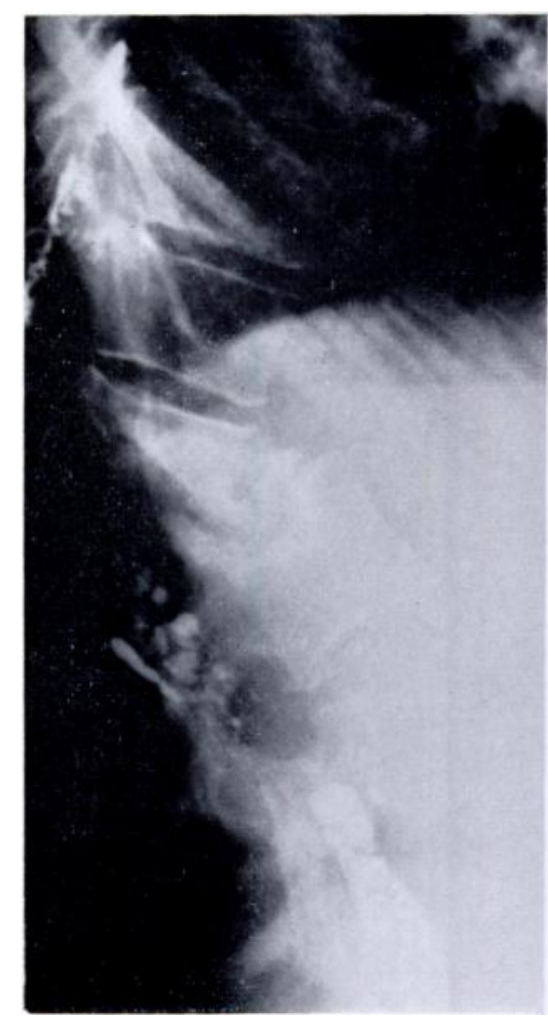

FIG. 15

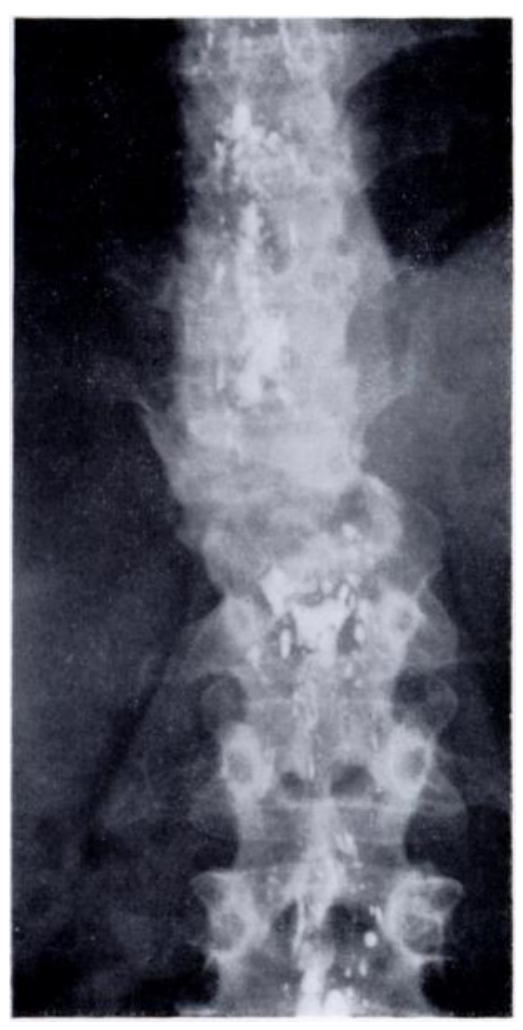

Fig. 16

Radiographs one year and eight months after operation. Figure 15-Lateral view. Figure 16-Antero-posterior view.

control of the bladder for almost two months, so the rapid improvement in the function of the spinal cord after the operation came as a most gratifying surprise.

It appears to have been a mistake to use cortical bone for bridging the gap. In another patient in whom a similar method of internal fixation was used, large blocks of bone from the posterior part of the iliac bones were employed for reconstruction of the spine after total removal of the seventh thoracic and partial removal of the sixth and eighth thoracic vertebrae (Stener 1971). Fifteen months after this operation radiography demonstrated incorporation of the iliac grafts. Cancellous bone is far superior to cortical bone for grafting (Burwell 1969). 


\section{SUMMARY}

1. A twenty-six-year-old woman was paraplegic because of a benign giant-cell tumour which had destroyed the body of the twelfth thoracic vertebra completely and the bodies of the eleventh thoracic and first lumbar vertebrae partially. The tumour had expanded into both pleural cavities and displaced the aorta forward and to the left. The extent and topography of the tumour were evaluated before operation by angiography. The function of the spinal cord had not been improved significantly by laminectomy. It was therefore decided to attempt extirpation of the tumour by removing all remaining parts of the three vertebrae involved.

2. After the removal of the tumour, only the spinal cord with the thecal sac bridged the gap between the tenth thoracic and second lumbar vertebrae. The gap was bridged with struts of cortical bone from both tibiae and with two strong plates, all secured with steel wire. The metal was removed five months later because it had become loose. Gradual shortening and angulation of the spine then occurred, together with progressive resorption of the cortical grafts. Nevertheless, the operation restored the function of the spinal cord; the patient regained ability to walk and full control of the bladder.

We are grateful to Professor Tormod Hauge, Oslo, for sponsoring the operation and for providing facilities. Our thanks are due to Professor Carl Hirsch, Stockholm, for encouragement and advice. The help of the anaesthetist, Dr Liv Refsum, was invaluable. We are also indebted to Dr Ole Styri for assistance during part of the operation, and to Dr Arne Engeset for permission to publish the radiographs.

\section{REFERENCES}

BLrWtLl, R. G. (1969): The Fate of Bone Grafts. In Recent Advances in Orthopaedics, p. 115. Edited by A. G. Apley. London: J. \& A. Churchill Ltd.

Rflton, J. E. S., and Hall, J. E. (1967): An Operation Frame for Spinal Fusion. A New Apparatus Designed to Reduce Haemorrhage During Operation. Journal of Bone and Joint Surgery, 49-B, 327.

SteNfR, B. (1971): Total Spondylectomy in Chondrosarcoma Arising from the Seventh Thoracic Vertebra. Journal of Bone and Joint Surgery, 53-B, 288. 